\title{
ESPACIOS POLÍTICOS Y ESTILO ALTO-BAJO. UN ANÁLISIS DE DIRIGENTES PROVINCIALES, A PARTIR DE JUICIOS DE EXPERTOS en Argentina 2015*
}

\author{
Political Spaces and High-Low Style. An Analysis of Provincial \\ Leaders, Based on Expert Judgments in Argentina 2015
}

\section{DIEGO REYNOSO}

CONICET-Universidad de San Andrés, Argentina

\section{GONZALO VRONKISTINOS}

Universidad de San Andrés, Argentina

\begin{abstract}
RESUMEN
En este artículo se estima la posición de los principales dirigentes provinciales según su estilo Low (bajo o informal-popular) o High (alto o sobrio-refinado), en la terminología de Pierre Ostiguy. Para lograr eso se realizó una encuesta en cada una de las provincias a expertos en política provincial. En total se consultaron a 191 expertos. El trabajo ofrece la estimación promedio de 117 políticos provinciales, así como el error estándar de esas estimaciones, y se pone a prueba la hipótesis acerca de que las diferencias en el estilo están asociadas a la pertenencia a un espacio político. En otras palabras, que los políticos panperonistas tienden a tener un estilo Low y los no peronistas, y en especial los de Cambiemos, un estilo High.
\end{abstract}

Palabras clave: Alto-Bajo, estilo político, política subnacional, encuesta, peronismo.

\begin{abstract}
This article estimates the position of the main provincial leaders according to their political style, "Low" (informal-popular) or "High" (sober-refined), in terms of Pierre Ostiguy's framework. To achieve this, a expert-survey was carried out in each province. 191 experts were consulted. The work offers the average estimate of 117 provincial politicians, as well as the standard error of those estimates, and tests the hypothesis that differences in style are associated with the leaders's political space. In other words, Peronist politicians tend to have a "Low" style and non-Peronist ones, and especially those of Cambiemos, a "High" style.
\end{abstract}

Keywords: High-Low, political style, sub-national politics, polling, Peronism.

\footnotetext{
La investigación contó con el apoyo financiero del Consejo Nacional de Investigaciones Científicas y Técnicas y de la Universidad Nacional de La Matanza a través de los proyectos PIP-CONICET 0838-15 y PROINCE-UNLaM DS042. Los autores agradecen a dos revisores anónimos y a Pierre Ostiguy por sus generosos comentarios y sugerencias.
} 


\section{INTRODUCCIÓN}

Existe consenso respecto de la dificultad de traducir las dimensiones de competencia partidista europeas a las coordenadas latinoamericanas, en general, y argentina en particular (Di Tella 1972, 2015). En el caso argentino, las posiciones político-ideológicas de uno de los principales partidos políticos, el Partido Justicialista (PJ), se vieron trastocadas en los años 90, cuando durante el gobierno del presidente Carlos Menem se dio un giro de 180 grados en las orientaciones históricas que este partido había sostenido. En esos años, el PJ pasó de impulsar políticas de tipo proestado y protrabajador a impulsar políticas de tipo promercado y proempresarios (Stokes 2001). Sin embargo, pese a este giro ideológico, no mutó la composición de su coalición social, es decir: siguió teniendo un fuerte respaldo de los sectores populares (Mora y Araujo 2011). En el año 2003, el Partido Justicialista (PJ) volvió al poder de la mano de Néstor Kirchner primero (2003-2007) y continuó con Cristina Fernández de Kirchner (2007-2015). En este nuevo período de gobierno, la coalición Frente para la Victoria, que integró el PJ, impulsó políticas proestado y protrabajo manteniendo al mismo tiempo el apoyo de los sectores populares (Mora y Araujo 1985, 2011). Algo similar se puede sostener de las oscilaciones ideológicas de la Unión Cívica Radical (UCR) si se comparan la orientación keynesiana y socialdemócrata del gobierno de Raúl Alfonsín (1983-1989) con la orientación de corte liberal-conservador del gobierno de Fernando De La Rúa (1999-2001), así como las que impulsa la coalición Cambiemos del gobierno de Mauricio Macri (2015-2019). Incluso con esas oscilaciones, las coaliciones que integra la UCR mantienen el respaldo de los sectores medios y medio altos de la sociedad (Mora y Araujo 1985, 2011).

La experiencia de estos años sugiere que no parece importar la orientación política o ideológica del partido de gobierno. Cualquiera sea ésta, el respaldo y el apoyo de la coalición social se mantienen. Como señalan atinadamente en su estudio Kitschelt y Wilkinson (2007), los partidos latinoamericanos no son exclusivamente identificables en la dimensión izquierda-derecha sino que se diferencian mediante el cultivo de redes clientelares como estrategia alternativa de identificación y lealtad entre éstos y los votantes. Otros trabajos, destacan la existencia de identidades sociales y culturales que organizan el espacio entre un campo popular o otro campo más elitista, independientemente del contenido ideológico de las políticas públicas que impulsen. En ese marco, Pierre Ostiguy sostiene que existen elementos culturales y comportamentales que se activan políticamente en muchos sistemas de partidos de Latinoamérica (Ostiguy 2009a), y en especial de Argentina (Ostiguy 2009b). Se trata del estilo y la estética socio-política y socio-cultural de los candidatos que opera como un vínculo representativo. Esta dimensión fractura el campo de competencia política en dos polos: por un lado, aquellos políticos que se destacan en el espacio público por su estilo "informal y coloquial popular", vinculado a las clases populares y/o bajas (el polo identificado con el término Low) y aquellos políticos que se destacan por su estilo "formal y sobrio", que se asocia a las clases medias altas y altas, o al de la elite (el polo identificado como el término High). 
Históricamente en Argentina, esta distinción parece tener raíces profundas: unitarios y federales, además de diferenciarse por sus posiciones en torno a la organización política del estado y la política comercial, también se diferenciaron por sus respectivas reivindicaciones estético-culturales. Sarmiento, en el XIX planteaba la caracterización de unitarios y federales, en términos peyorativos, como un conflicto entre la civilización y la barbarie (Sarmiento 2010). La historiografía argentina da cuenta de ello en las caracterizaciones que se hacen de unos y otros (Svampa 1994). Así, la identidad unitaria se conformó no solo a través de proyectos políticos y posturas doctrinarias, sino también por la pertenencia a un determinado orden social y económico. Los comportamientos culturales que solían identificarse con los unitarios como el bien vestirse, ir al café y otras prácticas que denotan cierto refinamiento y sofisticación" (Zubizarreta 2014). Siguiendo a Ostiguy (2009a), existen estrategias de posicionamiento de los dirigentes políticos que se materializan en estilos discursivos o de presentación pública, de acuerdo a los partidos de pertenencia y los apoyos sociales de éstos. Así, los dirigentes políticos identificados en las diferentes coaliciones panperonistas (kirchneristas o justicialistas) tenderán a adoptar estilos "populares informales" mientras que los políticos alineados con las diferentes coaliciones no peronistas, o aún más marcadamente antiperonistas, tenderán a adoptar estilos formales y sobrios. Esta expectativa respecto de los estilos políticos ¿refiere sólo a los modos de presencia pública de los líderes nacionales más representativos o es un sello distintivo partidario e identitario profundo? En otras palabras, nos preguntamos si los estilos que podrían cultivar los diferentes espacios políticos encuentran sus raíces profundas en el ámbito provincial. Al fin y al cabo, toda política es local.

En este trabajo ofrecemos una estimación del "estilo" de los principales dirigentes políticos provinciales argentinos, en total 117, que compitieron en las elecciones de gobernador de 2015 en las 24 provincias a partir de una encuesta que recoge el juicio de 191 expertos. También ofrecemos evidencia de la diferenciación significativa existente en cuanto al estilo de los candidatos, según las coaliciones o espacios políticos que integran. Finalmente, presentamos una comparación provincial según los niveles de diferenciación en las dos dimensiones de los principales candidatos a la gobernación, esto es: la ideológica y el estilo.

Sostenemos, en primer lugar, que los candidatos se agrupan en coaliciones o espacios políticos nacionales que presentan cierto grado de diferenciación en cuanto a sus estilos, lo que respalda en buena medida la hipótesis de Ostiguy. En otras palabras, existe una tendencia clara de estilo Low entre panperonistas, por un lado, y de estilo High entre no-peronistas y antiperonistas, por el otro. En segundo lugar, observamos dirigentes que buscan diferenciarse en la competencia cultivando un estilo Low, que se desvía de sus espacios políticos de pertenencia. En tercer lugar, y como consecuencia de la diferenciación individual, existen provincias en donde se observan relativas "anomalías" entre el espacio político y el estilo de los candidatos. 


\section{ESTADO DE LA CUESTIÓN SOBRE LOS ESPACIOS POLÍTICOS ARGENTINOS Y EL ESTILO POLÍTICO}

El estudio de los espacios políticos de la competencia es un tema clásico de la ciencia política contemporánea. Desde las primeras teorizaciones de Anthony Downs (1957) sumada a las inteligentes críticas que recibió (Stokes 1963), las teorías formales fueron mejorando las herramientas de los modelos (McKelvey y Ordeshook 1976; Brams 1983; Enelow y Hinich 1984; Shepsle y Cohen 1990). Junto a estas contribuciones, en el campo de la política comparada se indagaron los espacios de competencia empíricamente en las democracias más longevas, donde la dimensión ideológica clásica izquierda-derecha permitía ordenar y clasificar a los sistemas de partidos (Inglehart y Klingemann 1976; Sani y Sartori 1983; Castles y Mair 1984; Huber 1989; Knutsen 1988, 1989; Huber e Inglehart 1995). En ese mismo campo de estudio se han realizado investigaciones comparadas sobre los espacios políticos de competencia en Latinoamérica (Coppedge 1998, 2007; Colomer y Escatel 2005; Rivas 2006; Alcántara y Rivas 2007; Alcántara 2008; Saiegh 2009, 2015; Kitschelt et al. 2010; Wiesehomeier 2010; Wiesehomeier y Doyle 2012).

Todos los estudios coinciden en indicar que la dimensión ideológica clásica de izquierda-derecha permite ordenar a los partidos políticos argentinos, pero es incompleta. Junto a ésta, el sistema de competencia admite al menos otra u otras dimensiones adicionales. Recientemente, Reynoso (2018) ofrece evidencia respecto de la constitución de un espacio bidimensional organizado por la dimensión ideológica izquierda-derecha y por una dimensión adversarial (kirchnerismo vs antikirchnerismo) como especialmente relevante para analizar el espacio político de competencia que se cristalizó a nivel nacional y provincial en las elecciones de 2015. Otros trabajos también han destacado una dimensión similar de "adversariedad" entendiéndola como oficialismo-oposición (Zelaznik 2001). Esta dimensión, si bien por definición estructura las opciones políticas, considera las opciones como estrictamente funcionales. Como consecuencia, deja elementos de la pura aversión hacia algo o alguien fuera del análisis.

Pierrre Ostiguy (2009a) ha sostenido que, junto al clivaje ideológico, es necesario incluir una línea de división de tipo estético cultural con dos componentes o indicadores: uno de tipo socio-cultural y otra de tipo político-cultural. Esta dimensión o línea de división está vinculada a los estilos políticos y los modos de actuar en la escena política, y no tiene correspondencia necesaria con la ubicación ideológica de los partidos y los políticos individuales. Así, esta diferenciación político-social puede activarse (o no) en determinadas coyunturas críticas y volverse relevante a la hora de analizar el espacio político de competencia. Esta activación de una serie de marcadores estilísticos y del comportamiento en la autopresentación de los candidatos, junto a un adicional componente antielite, antiestablishment, fungen como un indicador potencial de los aspectos performativos del discurso populista (Ostiguy 2017; Casullo 2019), no exclusivo de América Latina (Mudde y Rovira Kaltwasser 2017). Dejaremos para otro 
trabajo la cuestión del populismo in toto y consideraremos el estilo como un position issue analíticamente discernible.

El componente socio-cultural de esta dimensión tiene sus indicadores en las distintas maneras, modales, modos de hablar y vestir y gustos exhibidos en público por parte los políticos (Ostiguy 2009a). En este sentido, separa a los políticos con estilos y apelaciones más populares, llanas o "plebeyas", de aquellos más refinados, "elevados" o intelectuales (Vommaro y Morresi 2016). En su componente político-cultural los indicadores provienen de las formas de liderazgo político y el modo de toma de decisiones. Se pueden diferenciar los políticos con comportamientos o declaraciones más institucionales, procedimentales e impersonales, de aquellos que poseen liderazgos personalistas y "fuertes", con actitud, firmeza y "coraje" (Ostiguy 2009a).

A partir de estas diferenciaciones se pueden agrupar, por un lado, en el polo High o "alto", a los políticos que poseen modales refinados, sobrios y que gustan presentarse como respetuosos de las instituciones formales y legalistas en la toma de decisiones. Por el otro, en el extremo Low o "bajo", a los políticos que poseen un modo más personalista de tomar decisiones, con un liderazgo fuerte y con un estilo directo, sentimental e informal con "su pueblo". De manera sucinta, resume Ostiguy:

"The first component of the high-low axis is the social-cultural appeal in politics. This component has to do with manners, demeanors, ways of speaking and dressing, and tastes displayed in public. On one pole, people publicly present themselves as well behaved, proper, composed, and perhaps even bookish. Negatively, they can appear as stiff, rigid, serious, colorless, somewhat distant, and boring. On the other pole, people frequently use a language that includes slang or folksy expressions and metaphors, are more demonstrative in their bodily or facial expressions as well as in their demeanor, and display more raw, culturally popular tastes. Politicians on the low are capable of being more uninhibited in public and are more apt to use coarse or popular language. They often appear-at least to the observer on the high-as more "colorful". While one may wish to call this dimension class-cultural, I have found that it is empirically most closely correlated, though not synonymous, with educational level. Although concrete sociocultural differences exist in all societies, these differences are usually not attached to political identities. In some cases, however, sociocultural differences do become politicized, in that cultural patterns such as manners, declared tastes, language and mode of behavior in public become associated with political identities or political choice" (Ostiguy 2009a: 7-8).

Como resultado de la interacción entre izquierda-derecha y alto-bajo, el espacio político de competencia queda comprendido en cuatro cuadrantes, en los que es posible ubicar a los actores políticos. La utilidad de este espacio bidimensional "radica en su capacidad explicativa de ciertos fenómenos a priori para- 
dójicos" (Alessandro 2009: 9). Es por eso que algunos estudios han indagado sobre la estructuración de algún aspecto de esta dimensión en la política local: Neuquén (Casullo y Pasetto 2017), CABA (Alessandro 2009), pero es aún muy poco lo que se ha producido a nivel subnacional comparado, por no decir que es inexistente.

La hipótesis de trabajo de Ostiguy es clara: además de la clásica dimensión ideológica que supone el espacio izquierda-derecha, existe una dimensión estética de estilos High-Low de tipo sociopolítica - cultural que organiza la oferta política de las coaliciones políticas nacionales y que eventualmente tiene anclaje en el ámbito provincial, conjeturamos nosotros.

En este sentido, en el siglo XIX la división entre Federales-Unitarios (además de las otras divisiones políticas y económicas) puede entenderse, en parte, como una confrontación de esos estilos culturales. En este marco, los partidos pueden posicionarse ideológicamente en la dimensión clásica izquierda-derecha, pero esta ubicación no es estética y puede ser coyuntural. Así, la UCR oscila entre el centro izquierda y el centro derecha, del mismo modo que el PJ. Sin embargo, lo que quedaría fijo o estructurado, en esas oscilaciones ideológico partidarias, es la ubicación en la dimensión high-low o elite-popular. De este modo, los peronistas organizados en el campo nacional-popular, en general tenderían a ubicarse en posiciones Low, mientras que los políticos más antiperonistas, tenderían a ubicarse en posiciones High.

\section{METODOLOGÍA}

Para estimar los estilos políticos de los principales políticos provinciales se recolectaron y analizaron las opiniones de jueces o "expertos" en cada provincia. La metodología de juicios de expertos tiene una larga tradición en la disciplina en general y en particular para la estimación de posiciones ideológicas (Laver y Hunt 1992; Budge 2000; King et al. 2003; Benoit y Laver 2007; Steenbergen y Marks 2007). Las encuestas a expertos han sido utilizadas en el análisis de los sistemas de partidos europeos (Castles y Mair 1984; Huber y Inglehart 1995; Laver 2001; Mair 2001), así como también sobre partidos y políticos de América Latina (Kitschelt et al. 2010; Wiesehomeier y Doyle 2012; Saiegh 2015).

Si bien Tetreault y Ostiguy (2014) han desarrollado una operacionalización cualitativa del estilo político para el caso argentino, nosotros aplicamos la metodología de estimación mediante juicios de expertos, ampliamente utilizada en la disciplina. Consideramos que el juicio de expertos presume un alto grado de validez en la respuesta y, además, es un mecanismo útil dado que las expresiones y percepciones de los expertos son claras y comprensibles a la hora de procesar los datos (Saiegh 2009). Aunque el uso de juicios expertos para realizar estimaciones y hacer comparaciones puede ser objeto de críticas, es cada vez más ampliamente aceptado en la comunidad académica, incluso obteniendo resultados más precisos (Benoit y Laver 2007) y por su utilidad para estimar fe- 
nómenos de los que no disponemos mediciones objetivas por su confiabilidad y eficiencia (Schedler 2012).

\section{Muestra}

Para la selección de la muestra de expertos, procedimos a contactar primero a aquellos expertos que pertenezcan a la carrera de investigador científico del CONICET o de la comisión de ciencia provincial en el área de ciencia política, sociología, etc. y que tuvieran publicaciones vinculadas con la política subnacional. Los contactos se establecieron mediante dos modalidades: vía correo electrónico, en primera instancia, o vía telefónica, en una segunda instancia. En general, la muestra no la integran expertos pertenecientes a una misma institución académica. El total de expertos entrevistados fue de 191, repartidos en las 24 provincias. Los 191 expertos respondieron a todas las preguntas del cuestionario para los 14 estímulos nacionales (políticos nacionales) que se les pidió evaluar y clasificar. Para la clasificación de los 117 estímulos provinciales se dividió la muestra de expertos en 24 submuestras (a nivel provincial), cada una de las cuales evaluó en promedio a 5 políticos de la provincia en cuestión ${ }^{1}$.

\section{Instrumento de medición}

Mediante el envío de un cuestionario vía correo electrónico, los 191 entrevistados clasificaron a 14 políticos nacionales en diferentes dimensiones de competencia (ideología, estilo político, proximidad con el gobierno nacional y liberalismo). En todos los casos fueron utilizadas escalas de 1-7 puntos, donde 1 representaba un extremo de la dimensión y 7 el extremo opuesto. La pregunta efectuada para determinar el juicio del experto sobre el estilo político de un dirigente fue la siguiente:

Habitualmente los políticos tienen diferentes formas de expresar y/o comunicar sus ideas al público. En una escala de 1-7 puntos, donde 1 significa que utiliza en extremo un "estilo informal y un lenguaje popular" y 7 significa que utiliza en extremo un "estilo sobrio, refinado y un lenguaje más formal" ¿Dónde situaría usted a los siguientes actores o líderes políticos?

Una vez que los entrevistados clasificaban a cada uno de los políticos nacionales, se les preguntaba la provincia de residencia y trabajo, de modo que ello permitía filtrar el próximo conjunto de preguntas delimitándolo solo a los políticos

En todas las provincias se enfrentaron en las elecciones al menos un candidato del Frente para la Victoria (FPV) y de Cambiemos, junto a otros candidatos del Frente Renovador, del Frente de Izquierda y de los Trabajadores (FIT) y algún candidato provincia (como por ejemplo el Movimiento Popular Neuquino). En este sentido, en promedio cada experto efectuó juicios para ubicar en las escalas a 5 políticos de su provincia (Reynoso 2018). 
de la provincia en cuestión. A continuación, los entrevistados respondían las mismas preguntas para un conjunto reducido de 4 a 6 dirigentes provinciales. Los políticos escogidos fueron el gobernador, los candidatos a gobernador de las principales coaliciones gravitantes en la provincia y, en algunos casos considerado relevante, se incluyó al intendente de la ciudad capital de la provincia ${ }^{2}$.

Ex post a la recolección de los datos, los políticos provinciales fueron ubicados, según el partido de procedencia, en alguna de las coaliciones políticas nacionales que participaron en la elección presidencial de 2015. Se tomó en cuenta para ello la lista en la que se presentaron (en caso de los candidatos a gobernador) y, en los casos en que ello no hubiera sido suficiente o no reflejara con claridad su alineamiento nacional, indagamos mediante a consultas a informantes y expertos locales. De este modo, pudimos clasificar a los políticos en 5 espacios o coaliciones políticas, de los cuales el Frente para la Victoria (FPV) y Cambiemos constituyen las más grandes en cuanto a adscripciones debido a su implante territorial, seguidos del Frente Amplio Progresista (FAP), Frente Renovador-Una Nación (FR) y el Frente de Izquierda y los Trabajadores (FIT). Un conjunto residual de gobernadores y candidatos perteneciente al PJ, pero que no se alinearon en el FPV, fueron reunidos junto a los que integraban el conjunto Frente Renovador (FR), a los que definimos como PJ-noK. La Tabla 1 presenta el número de políticos que fueron agrupados en los diferentes bloques. Agregamos una sexta categoría residual (otros) para agrupar a aquellos que incluso con un segundo análisis no podían ser clasificados en los bloques nacionales, como el Movimiento Popular Neuquino (Neuquén), Fuerza Republicana (Tucumán) o Autodeterminación y Libertad (CABA).

Tabla 1. Resumen de variable 'estilo político' según espacio político.

\begin{tabular}{crrrrrr}
\hline Espacio & \multicolumn{1}{c}{ media } & \multicolumn{1}{c}{ mediana } & \multicolumn{1}{c}{ desvio } & \multicolumn{1}{c}{$\min$} & \multicolumn{1}{c}{$\mathrm{Nax}$} \\
\hline FPV & 3.258 & 3.2 & 0.9229 & 1 & 5.143 & 56 \\
Cambiemos & 4.001 & 4.222 & 1.219 & 1.111 & 6 & 41 \\
PJ-noK & 3.299 & 3.277 & 0.5842 & 2.2 & 4.4 & 16 \\
FAP & 4.629 & 4.778 & 0.5594 & 3.778 & 5.268 & 5 \\
FIT & 3.364 & 3.125 & 0.5464 & 2.75 & 4.25 & 7 \\
Otros & 3.958 & 4.062 & 0.6539 & 3.167 & 4.7 & 6 \\
\hline
\end{tabular}

Si bien la dimensión alto-bajo implica un conjunto de aspectos del orden estético de los candidatos ("maneras, modales, modos de hablar y vestir y gustos exhibidos en públicos"), la pregunta que escogimos como indicador del estilo remite sólo al modo de hablar y a la sencillez/sofisticación del uso del lenguaje. Desde luego, una tentativa de medición más integral debería incluir indicadores de vestimenta y gustos, más difíciles de precisar en un cuestionario. De todos modos, dejamos para la siguiente ola del estudio la posibilidad de ampliar la medición. 
La Tabla 1 indica que el FPV, con una media $=3.26$ y una mediana $=3.20$, es el partido que en promedio tiene dirigentes cuyo estilo es el más inclinado al polo Low de la escala. Casi en la misma posición, con una media $=3.30$ y mediana $=$ 3.28, se ubican los dirigentes del Pj-noK (Peronismo no Kirchnerista). En contraposición, el espacio político con dirigentes que cultivan un estilo más High es el FAP, con una media estimada de 4.63 y una mediana de 4.78. Cambiemos presenta una estimación media $=4.00 \mathrm{y}$ una mediana $=4.22$, ubicándose en promedio del lado High de la escala. Nótese sin embargo, que en Cambiemos existen 4 dirigentes outliers para el estilo medio del espacio político. Para tener una idea más clara de lo que representan los valores que describen la Tabla 1 presentamos en la figura 3 una visualización de la distribución de la variable clave de estudio según los espacios políticos de pertenencia.

\section{RESULTADOS}

La estimación de la posición de cada político en cada dimensión surge del promedio de las clasificaciones de los expertos, de acuerdo a una práctica ya clásica en la disciplina. Asumimos que los políticos ocupan una posición en cada una de las dimensiones y que esas posiciones pueden ser estimadas a partir de las percepciones de los expertos entrevistados. Cada una de esas percepciones se transforman en un valor en una escala discreta de 1-7 puntos e inferimos que la posición de los políticos en esa escala puede estimarse a partir del promedio de las clasificaciones que los expertos realizan. Cuanto menor sea la diferencia entre las clasificaciones que los $n$ expertos vierten sobre un político $j$, más precisa será nuestra estimación, y viceversa. De este modo, asumimos que las percepciones de los expertos (transformadas en una medida de posicionamiento) son buenos indicadores de las posiciones relativas de los políticos en cada dimensión. La figura 1 visualiza las estimaciones medias de cada político, así como el error estándar de tales estimaciones, por parte de los expertos.

Los políticos ubicados en los extremos de la distribución son: en el polo Low, el ex gobernador de San Juan, Jose Luis Gioja, con una estimación de 1 y un error estándar de la estimación igual a 0; en el extremo highde la distribución se encuentra el senador por Formosa Luis Naidenoff, cuya estimación media es de 6 puntos con un error estándar de 0.365. La figura 1 identifica, además, a los diferentes políticos según el espacio político al que adscribía en la elección de 2015. En general, los políticos del FPV son mayoría en el extremo Low de la escala, aunque destacan con valores muy bajos 4 políticos pertenecientes a Cambiemos: Miguel del Sel (Santa Fé), Luis Juez (Córdoba), Alfredo de Angelis (Entre Ríos) y Alfredo Olmedo (Salta). En el extremo High predominan los políticos de Cambiemos, aunque figuran tres excepciones: Miguel Angel Pichetto 
Figura 1. Estimación media y error estándar de 131 políticos nacionales y provinciales, a partir de juicios de expertos.

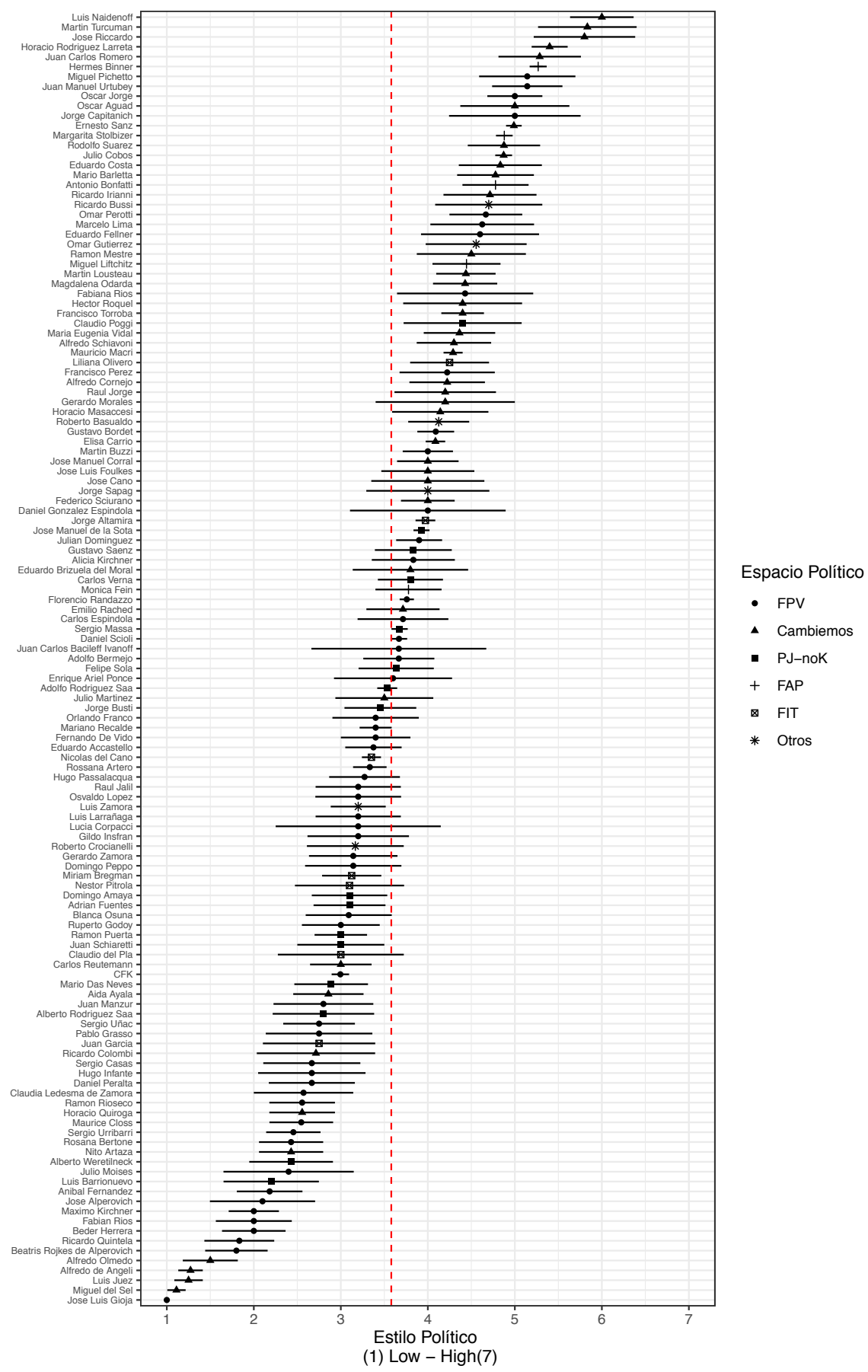

Los puntos indican el promedio de los juicios de entrevistados respecto de un político específico (eje vertical), y las líneas indican el error estándar de los juicios en torno al político particular (i.e. la dispersión). La escala va de 1, el valor más bajo posible, a 7, el valor más alto. 
(Río Negro), Juan Manuel Urtubey (Salta) y Jorge Capitanich (Chaco), los tres pertenecientes al $\mathrm{FPV}^{3}$.

En general se puede apreciar una alta consonancia entre los expertos a la hora de emitir sus juicios respecto del estilo de los políticos evaluados. El promedio de los desvíos estándar de los juicios es de 1.3 y el máximo alcanzado de los desvíos es de 2.65. La figura 2, por su parte, presenta la relación entre la estimación del estilo del político y el desvío estándar de la estimación, o, en otras palabras, el desacuerdo entre los expertos acerca de cómo calificarlo. En general, se aprecia una tenue relación en forma de u invertida entre el valor estimado del estilo y el desvío estándar. Ello implica cierta heterocedasticidad en la estimación o que hay más convergencia en los juicios de los expertos respecto de los políticos cuyos estilos son más extremos.

En el siguiente apartado trataremos de someter a una prueba estadística, de manera sistemática, la hipótesis de Ostiguy. Esto es, si las diferencias en el estilo son un elemento distintivo de los dirigentes que pertenecen a diferentes espacios políticos. $\mathrm{O}$, en otras palabras, si los políticos peronistas tienden a tener un estilo Low y los políticos no-peronistas o antiperonistas un estilo High.

La media promedio del estilo de los dirigentes clasificados es de 3.58 y la mediana de 3.66. La tabla 1 presenta los valores de media, mediana y demás estadísticos resúmenes, que describen la variable estilo, según el espacio político al que pertenecen los dirigentes clasificados.

\footnotetext{
Tanto en el caso del senador por Rio Negro, Miguel Angel Pichetto, como en el del gobernador de Salta, Juan Manuel Urutubey, con posterioridad a la coyuntura del 2015 pasaron a engrosar las filas del Peronismo no Kirchnerista; en cierto modo, parte de sus diferencias con el espacio de la expresidenta Cristina Fernandez han sido "las formas". En otras palabras, una cuestión de estilo. Como elemento en favor de la relevancia del estilo como expresión de una identidad, en la coyuntura de la elección presidencial de 2019, el senador por Rio Negro terminó siendo candidato a vicepresidente en la fórmula presidencial de Cambiemos (Juntos por el Cambio), la coalición de estilo "high". En un sentido similar, el gobernador de Salta fue candidato a vicepresidente por otra coalición electoral, pero también fue invitado a integrar la coalición de Juntos por el Cambio.
} 
Figura 2. Estilo Político estimado y desvío estándar de los juicios expertos.

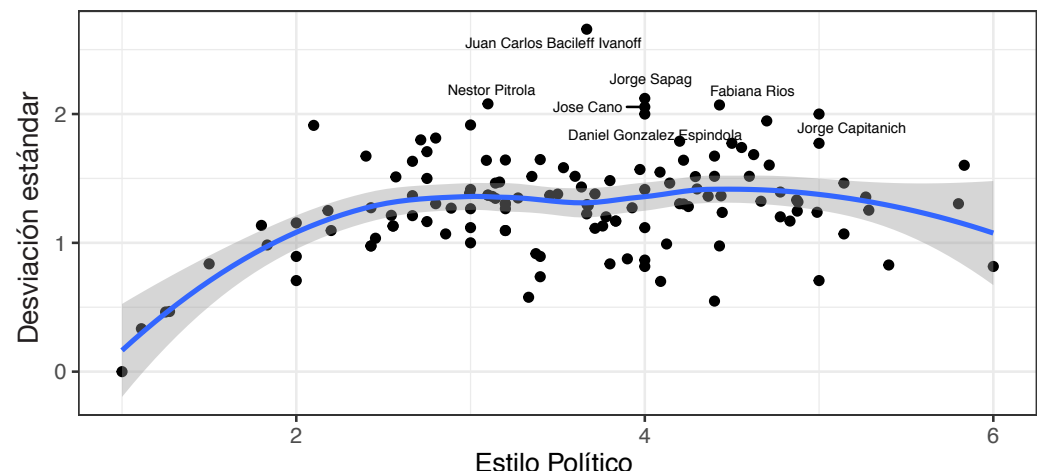

(1) Low - High (7)

La figura permite relacionar la dispersión de los juicios (el desacuerdo entre los expertos) y la estimación obtenida respecto del estilo de los candidatos. De este modo, observamos que en los extremos los juicios se desvían menos y en el centro los desvíos son mayores. Ello podría sugerir que los políticos con valores promedios medianos, podrían ser producto del desacuerdo de los expertos respecto de donde ubicarlos, más que de su propia posición. En esos casos, las estimaciones con desvíos superiores a 1.96 (cuyos nombres parecen en la figura) podrían cuestionarse. No obstante, el rango de los desvíos es aceptable.

Figura 3. Distribución de estilo de políticos provinciales según espacio político.

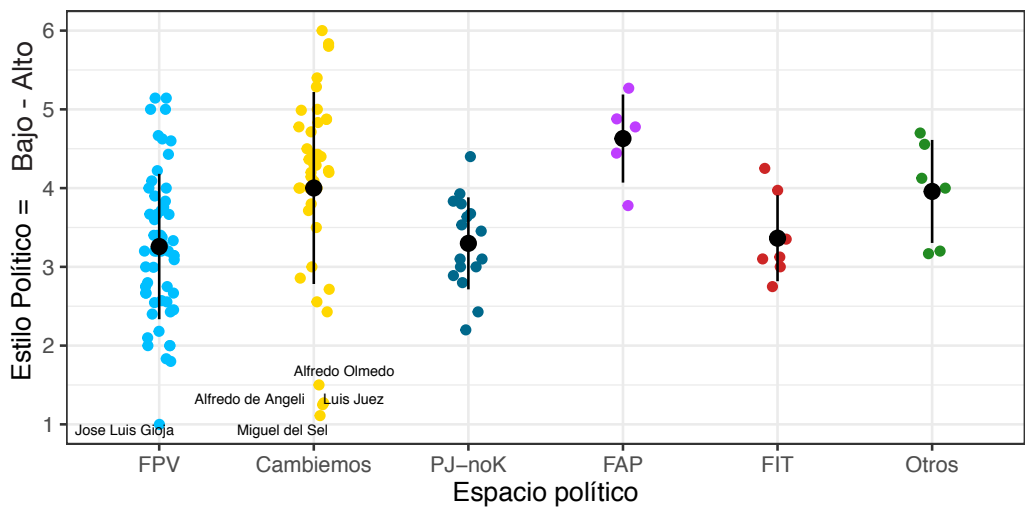

La Figura permite observar comparativamente la distribución de los estilos de los políticos de un espacio, frente a la distribución de otro espacio. Se observa que la distribución del FPV presenta una media más baja que la de Cambiemos y el FAP. Por otra parte, se puede apreciar los 4 dirigentes de Cambiemos que tienen posiciones outliers respecto de su espacio

Como se puede observar en la figura 3, sobresalen dentro de la distribución de Cambiemos el dirigente salteño Alfredo Olmedo (identificado por el uso de su campera amarilla), el dirigente rural de Entre Ríos Alfredo De Agelis, el diputado por Córdoba Luis Juez (experonista) y el excandidato a gobernador de 
Santa Fe Miguel del Sel (un actor cómico). Se trata de 4 dirigentes outliers desde el punto de vista del estilo sobriedad que cultivan los dirigentes de Cambiemos, y que puede encontrar una explicación en el origen y procedencia de cada uno de ellos. Considerando las distribuciones del estilo de los dirigentes de los diferentes espacios políticos, realizamos un test de análisis de varianza, para determinar si existen diferencias en la distribución que puedan adjudicarse a la pertenencia a esos espacios políticos. En la Tabla 2 se presenta el resultado del test ANOVA para determinar si existen diferencias estadísticas entre los espacios políticos.

Tabla 2. Análisis de Varianza del Modelo

\begin{tabular}{lccccc}
\hline & Df & Sum Sq & Mean Sq & F value & $\operatorname{Pr}(>\mathrm{F})$ \\
\hline Espacio & 5 & 21.04 & 4.207 & 4.512 & 0.0008161 \\
Residuos & 125 & 116.6 & 0.9325 & NA & NA \\
\hline
\end{tabular}

Los resultados del análisis de varianza que presenta la tabla 2 arrojan un valor de la prueba $F=4.512$ y su correspondiente $p$-value $=0.0008161$ que permiten rechazar la hipótesis nula que considera que la pertenencia a un espacio político no está asociada al estilo. En este sentido, podemos asumir que existen diferencias promedios de estilo entre los espacios políticos analizados que son estadísticamente significativas. Desde luego, las diferencias entre los espacios políticos no son en todos los casos significativas. La Tabla 3 resume los $p$-value del $t$-test que resulta de la comparación de a pares entre espacios.

Tabla 3. Comparación de a pares de t-test

\begin{tabular}{lccccc}
\hline & FPV & Cambiemos & PJ-noK & FAP & FIT \\
\hline Cambiemos & 0.004111 & NA & NA & NA & NA \\
PJ-noK & 1 & 0.1789 & NA & NA & NA \\
FAP & 0.04003 & 1 & 0.1057 & NA & NA \\
FIT & 1 & 0.9825 & 1 & 0.2972 & NA \\
Otros & 0.9408 & 1 & 1 & 1 & 1 \\
\hline
\end{tabular}

En efecto, los resultados del $t$-test entre los espacios políticos confirman que entre el FPV y Cambiemos existen diferencias estadísticamente significativas (incluso considerando los outliers de Cambiemos), así como entre el primero y el más sobrio FAP. Esto significa que en ambas comparaciones de pares el $p$-value $<.05$, como puede observarse en la Tabla. Por otra parte, se confirma que entre el FPV y el Peronismo no-K no existen diferencias de estilo. En las demás comparaciones no se aprecian diferencias estadísticamente significativas. 


\section{DISCUSIÓN}

Las diferencias encontradas entre los principales espacios políticos que compitieron en todo el territorio en el año 2015, indican que el estilo expresa una identidad política. Estos hallazgos confirman, en general, la hipótesis de Ostiguy. Algunos peronistas, en particular los "kirchneristas" que integran el FPV, tienden a presentar un estilo expresivo más Low que, en promedio, los políticos de Cambiemos y del FAP, los cuales en general tienden a presentar un estilo más High.

Desde luego, un análisis más profundo a nivel de cada provincia permite una discusión más particular de los hallazgos generales. A tales efectos la figura 4 presenta el espacio de competencia de cada provincia de acuerdo a un par de ejes cartesianos, o dimensiones, que aquí consideramos relevantes: la ideológica en el eje horizontal, entendida como izquierda-derecha, y la del estilo en el eje vertical, entendida como alto-bajo o High y Low. En general, se observa un alineamiento ideológico claro: los dirigentes del FIT, en la izquierda, los del FPV en el centro izquierda, al igual que los dirigentes del FAP, los peronistas no kirchneristas en el centro y los dirigentes de Cambiemos en el centro derecha, consistente con lo que otros trabajos han demostrado (Reynoso 2018). Conjuntamente con el alineamiento ideológico, propiamente político, se puede apreciar la diferenciación no política basada en el estilo de los competidores. Así, es posible divisar en Buenos Aires a María Eugenia Vidal (Cambiemos) mucho más arriba y hacia la derecha de Aníbal Fernández (FPV-PJ), por ejemplo, de acuerdo a la expectativa teórica4

Pero no todos los casos particulares, replican de manera idéntica los patrones generales. Un caso singular que ilustra la cuestión es Río Negro. En un contexto de convergencia ideológica (según los juicios de expertos), esto es que los políticos no tienen grandes diferencias en términos de izquierda-derecha, el estilo político produce una mayor diferenciación entre los dirigentes y candidatos en competencia. Conjeturamos que se trata de una estrategia de identificación no basada en cuestiones de política pública (policy), capaz de romper con la indiferencia que los electores pudieran percibir en la dimensión izquierda-derecha. En la provincia destacan por contraposición dos actores provinciales claves: Miguel Angel Pichetto y Alberto Weretilneck. El primero, senador nacional desde el año 2001, se caracteriza por posturas atemperadas, en donde suele resaltarse, sobre todo en su conducta institucional en la Cámara de Senadores, la cordialidad y la cortesía. En cuanto a la vestimenta, acostumbra lucir de modo elegante y discreto (saco y corbata). En contraste, Weretilneck se sitúa en el polo Low, cultivando una estética cercana al elector rionegrino común. Con un discurso inclusivo, con referencias simples y expresiones claras, prefiere "achicar" la distancia con el pueblo y, según algunas declaraciones de expertos provinciales, "interpreta al rionegrino medio". De este modo se muestra relajado y utiliza la informalidad como elemento de vínculo con los ciudadanos. En este sentido, suele situarse

El posicionamiento de los dirigentes en la escala izquierda-derecha se obtuvo mediante la misma metodología y con los mismos expertos. 
Figura 4. Espacios de competencia provinciales, a partir de la intersección entre el estilo político (eje vertical) y el posicionamiento ideológico (eje horizontal).
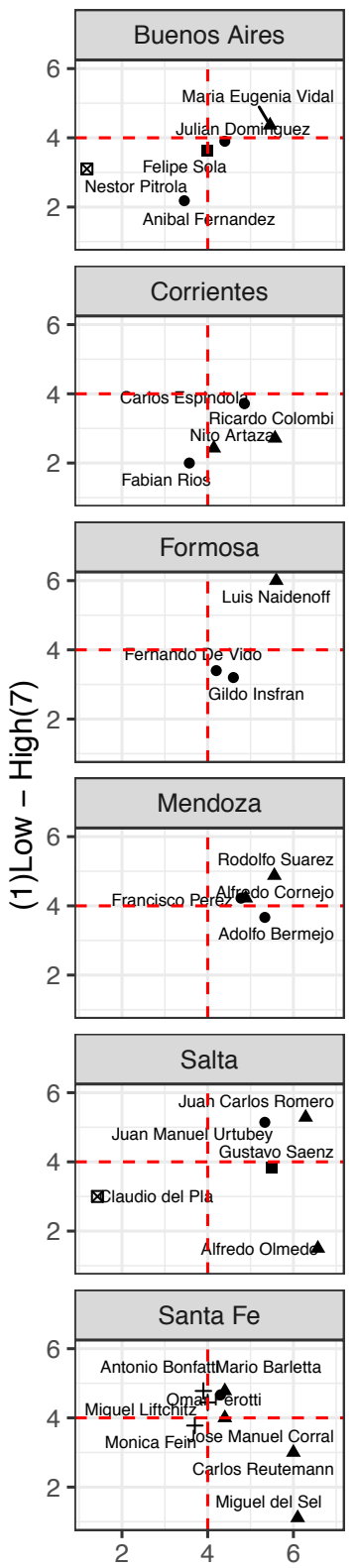
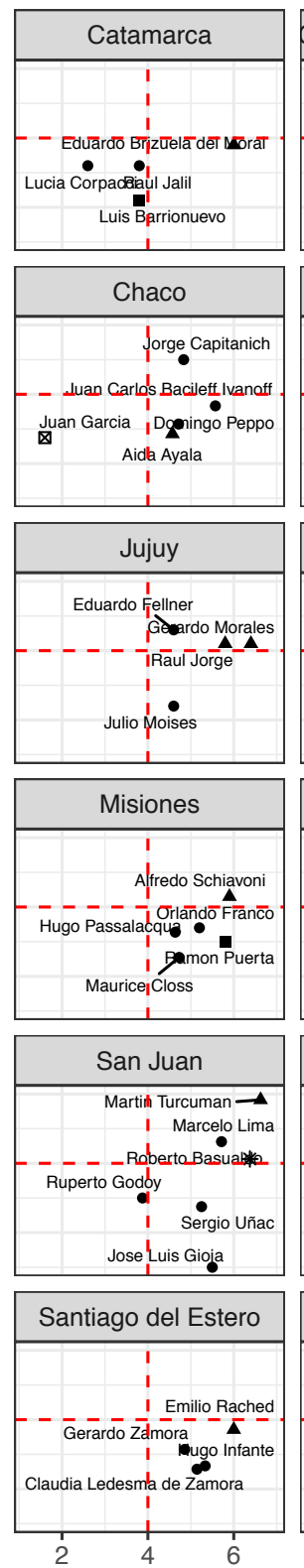
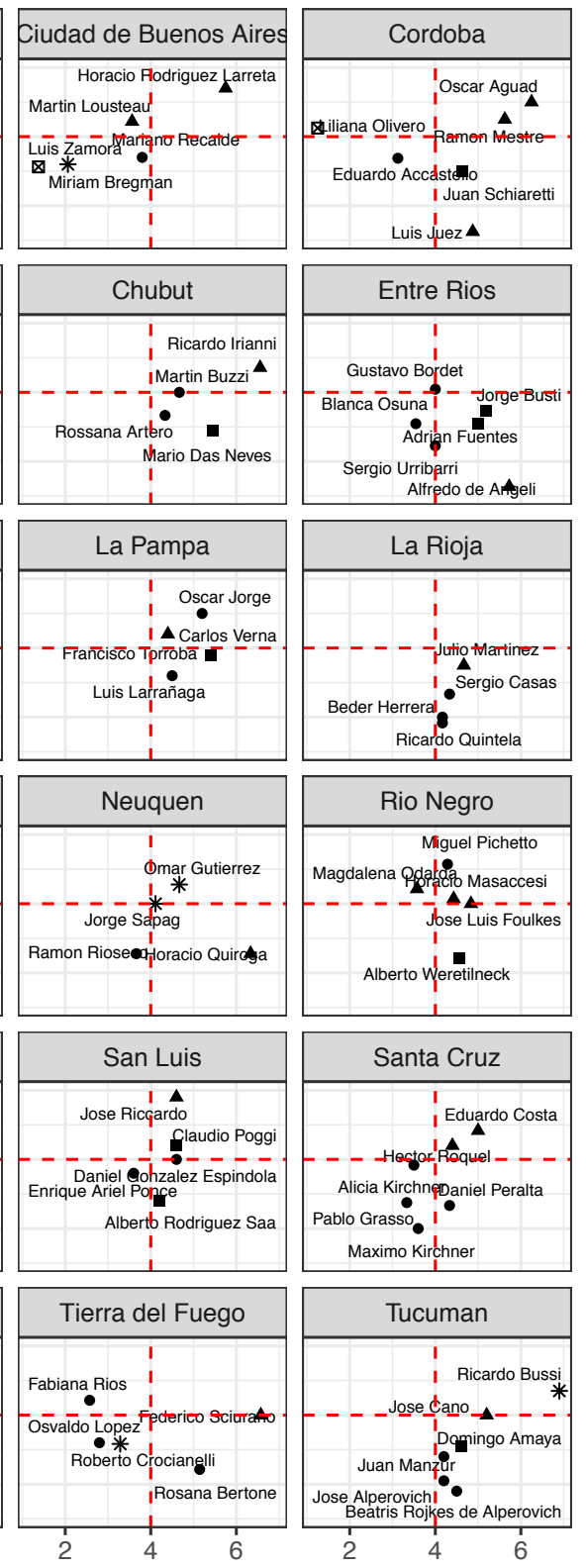

(1) Izquierda - Derecha(7)

Los espacios replican en su mayoría una orientación hacia la izquierda y hacia abajo, de los dirigentes del FPV o de los dirigentes del PJ-noK, y una orientación hacia la derecha y hacia arriba, de los dirigentes de Cambiemos, con la excepción de Córdoba, Entre Rios, Salta y Santa Fé, por un lado, y de Rio Negro, por el otro. 
discursivamente como uno más de la población, presentándose en discursos públicos y en recorridas por los barrios como "un hijo de un laburante", "no de un dueño de una multinacional", un vecino más de Río Negro.

La explotación del estilo como un recurso electoral, más allá del posicionamiento ideológico o como contrabalanceador de éste, encuentra en los casos de Salta, Córdoba, Santa Fe o Entre Ríos ilustraciones paradigmáticas, donde son los políticos de Cambiemos los que se diferencian de sus contrincantes por cultivar un estilo Low, más propio teóricamente de sus rivales.

Salta es un caso por demás ilustrativo. En esta provincia el estilo parece operar con mayor intensidad que la ideología, como en el caso de Alfredo Olmedo. En contraste a esta figura por demás destacada, los expertos consideran que los dos referentes peronistas provinciales cultivan un estilo High, como es el caso de Juan Carlos Romero, ex gobernador peronista, y Juan Manuel Urtubey, actual gobernador y candidato a vicepresidente. A diferencia de Urtubey, que conserva un estilo clásico de presentación y moderado en sus intervenciones, Alfredo Olmedo es nacionalmente reconocido por sus modos de expresarse en público y, sobre todo, de vestir. Este código de vestimenta audaz, relajado e informal es acompañado por su lenguaje sumamente coloquial, "de la calle", su tonada provincial al hablar y sus gestos, que lo acercan a los sectores populares de la sociedad salteña, pese a ser un reconocido propietario de campos. El contenido de su discurso se completa con la utilización de metáforas, anécdotas, ejemplos cotidianos y concretos.

Con mayor o menor intensidad es posible, entonces, apreciar distinciones que no se ajustan a la hipótesis del estilo como diferenciador político. En Córdoba, Luis Juez (FAP - Cambiemos) se ubica no sólo debajo de sus socios políticos, Oscar Aguad (Cambiemos) o Ramón Mestre (Cambiemos), sino también de su competidor Peronista, Juan Schiaretti. Y en Santa Fe, por último, a Omar Perotti (PJ) y Miguel Lifschitz (Socialismo) moderados y en el centro del escenario frente a un Miguel del Sel (Cambiemos) que se sitúa, según los expertos, en el extremo inferior derecho.

Desde luego, si bien en términos generales la pertenencia a un espacio político está asociada con una tendencia en el estilo de sus dirigentes, en términos provinciales, más casuísticos, el esquema general no se reproduce de manera lineal y directa en cada una de las dinámicas de la competencia, como Catamarca, por ejemplo. Como sostiene Escolar (2011), es posible que un modelo nacional homogéneo no ajuste cuando las fracturas en temas centrales a niveles comunitarios subsistan por un proceso de integración nacional no del todo exitoso, ya sea porque el consenso racional se logra subdividiendo al Estado y al "Demos" o porque la división administrativa que hizo el Estado nacional genere nuevas comunidades con sus dimensiones propias, como lo indica al respecto para el caso de Neuquén (Casullo y Pasetto 2017) o el de CABA (Alessandro 2009). En estas condiciones, las organizaciones subnacionales de los partidos tienen motivos para privilegiar o anteponer las dimen- 
siones de escala provincial (Leiras 2010). Desde luego, si estas consideraciones aplican a los elementos de orientación política de los partidos, como el posicionamiento izquierda-derecha o liberal-conservador, mucho más débil es el mecanismo que explica la relación entre el partido y el estilo personal e individual que un dirigente puede adoptar.

\section{CONCLUSIÓN}

Pese a que universalmente se conoce que izquierda y derecha reducen la complejidad y resumen, como "atajos informativos", un conjunto de ideas de manera eficaz, existe el planteo de cierta literatura que destaca la imperfección que posee este clivaje para capturar la esencia de la dinámica política argentina. En este marco, Pierre Ostiguy (2009b) afirma que este eje programático debe ser complementado con la intersección ortogonal de una fractura que está presente desde el siglo XIX: el estilo como dimensión estructuradora de las identidades partidarias. En este trabajo indagamos en qué medida hay una correspondencia entre las coaliciones políticas, o espacios políticos, nacionales y los estilos de los dirigentes políticos provinciales.

Una línea de investigación futura demanda complementar el presente análisis añadiendo otras dimensiones que podrían estar relacionadas con la distinción entre oficialismo y oposición local o, como sugieren el caso neuquino, rionegrino y cordobés, con la existencia de una fractura entre los políticos del centro/ nacionales y los de la provincia. En tal sentido, como sostiene Behrend (2011, 256), la política provincial es el resultado de la historia política, las tradiciones provinciales, las instituciones electorales y políticas, el comportamiento de las élites, la estructura socioeconómica, las dinámicas partidarias, los actores sociales, las relaciones intergubernamentales y el federalismo fiscal, entre tantas otras cuestiones.

Lo que en este trabajo logramos fue estimar el posicionamiento de los principales dirigentes nacionales y subnacionales de Argentina para poder establecer comparaciones interprovinciales, a partir del juicio de 191 expertos vertidos en escalas de 1 a 7 puntos. De esta forma, se situó a 131 dirigentes: 14 nacionales y 117 provinciales, repartidos en las 23 provincias y la Ciudad Autónoma de Buenos Aires. Tomando como referencia la ubicación de cada uno de los dirigentes, se estimó el estilo que cultiva cada uno de ellos en la escala High-Low.

A partir de esas estimaciones individuales, ofrecimos evidencia de que los candidatos se agrupan en coaliciones o espacios políticos nacionales que presentan cierto grado de diferenciación en cuanto a sus estilos, lo que respalda en buena medida la hipótesis de Ostiguy. En otras palabras, que hay relativa homogeneidad en los estilos de panperonistas, por un lado, y de no-peronistas y antiperonistas, por el otro. En segundo lugar, encontramos que si bien hay diferencias o variaciones provinciales entre los políticos y al interior de los espacios, la estructura del espacio de competencia se replica en cada contexto provincial. En tercer 
lugar, y como consecuencia de la diferenciación individual, encontramos algunas "anomalías" singulares dadas por dirigentes que buscan diferenciarse en la competencia cultivando un estilo Low, que se desvía de sus espacios políticos que colectivamente responden a un estilo High de maneras públicas. Los resultados de la investigación aún no logran dar una respuesta general y definitiva acerca de cómo se estructura el espacio de competencia política en las provincias y cómo se agrega ello nacionalmente, pero aporta evidencia parcial sobre una dimensión singular destacada por parte de la literatura, como lo es el estilo.

\section{REFERENCIAS}

Alcántara, Manuel. 2008. "La escala de la izquierda". Nueva Sociedad 217: 72-85.

Alcántara, Manuel y Cristina Rivas. 2007. "Las dimensiones de la polarización partidista en América Latina." Política y Gobierno XIV (2): 349-90.

Alessandro, Martin. 2009. "Clivajes sociales, estrategias de los actores y sistema de partidos: la competencia política en la Ciudad de Buenos Aires (1995-2005)." Revista de La SAAP 3 (4): 581-614.

Behrend, Jaqueline. 2011. "Política subnacional y democracia." Revista de La SAAP 5(2): 24960.

Benoit, Kennethy y Michael Laver. 2007. “Estimating Party Policy Positions: Comparing Expert Surveys and Hand-Coded Content Analysis." Electoral Studies 26(1): 90-107.

Brams, Steven. 1983. Spatial Models of Election Competition. Lexington: The UMAP expository Monograph Series.

Budge, Ian. 2000. "Expert Judgements of Party Policy Positions: Uses and Limitations in Political Research." European Journal of Political Research 37(1): 103-13.

Cas Mudde y Cristóbal Rovira Kaltwasser. 2017. Populism. A Very Short Introduction. New York: Oxford University Press.

Castles, Francis y Peter Mair. 1984. "Left-Right Political Scales: Some 'Expert' Judgments." European Journal of Political Research 12(1): 73-88.

Casullo, María Esperanza. 2019. ¿Por qué funciona el populismo?. El discurso que sabe construir explicaciones convincentes de un mundo en crisis. Buenos Aires: Siglo XXI.

Casullo, María Esperanza y Alejo Pasetto. 2017. "La génesis de la 'neuquenidad' como construcción política." Enclave Comahue 22: 147-62.

Colomer, Josep y Luis Escatel. 2005. "La Dimensión Izquierda-Derecha en América Latina." Desarrollo Económico 45(177): 123-36.

Coppedge, Michael. 1998. “The Dynamic Diversity of Latin American Party Systems." Party Politics 4(4): 547-68.

Coppedge, Michael. 2007. "Continuity and Change in Latin American Party Systems." Taiwan Journal of Democracy 3(2): 119-49.

Di Tella, Torcuato. 1972. "La búsqueda de la fórmula política argentina." Desarrollo Económico, Vol.11 (42): 599-639.

Di Tella, Torcuato. 2015. Coaliciones Políticas. La Argentina en perspectiva. Buenos Aires: El Ateneo.

Downs, Anthony. 1957. "An Economic Theory of Political Action in a Demoracy." The Journal of Political Economy 65(2): 135-50.

Enelow, James y Melvin Hinich. 1984. The Spatial Theory of Voting. An Introduction. Cambridge: Cambridge University Press.

Escolar, Marcelo. 2011. "Nacionalización, comunidad cívica y coordinación electoral. Problemas para la integración del sistema político en estados democráticos multinivel." Revista de La SAAP 5(2): 263-304. 
Huber, John. 1989. "Values and Partisanship in Left-Right Orientations: Measuring Ideology." European Journal of Political Research 17: 599-621.

Huber, John y R. Inglehart. 1995. "Expert Interpretations of Party Space and Party Locations in 42 Societies." Party Politics 1(1): 73-111.

Inglehart, Ronald y Hans-Dieter Klingemann. 1976. "Party Identification, Ideological Preference and the Left-Right Dimension Among Western Mass Public." Party Identification and Beyond. London: Wiley.

King, Gary, Christopher J L Murray, Joshua a Salomon y Ajay Tandon. 2003. "Enhancing the Validity and Cross-Cultural Comparability of Measurement in Survey Research." The American Political Science Review 97 (4): 567-83.

Kitschelt, Herbert y Steven Wilkinson. 2007. Patrons, Clients, and Policies: Patterns of Democratic Accountability and Political Competition. Cambridge: Cambridge University Press. .

Kitschelt, Herbert, Kirk a. Hawkins, Juan Pablo Luna, Guillermo Rosas y Elizabeth J. Zechmeister. 2010. Latin American Party Systems. Cambridge: Cambridge University Press.

Knutsen, Oddbjorn. 1988. "The Impact of Structural and Ideological Party Cleavages in West European Democracies: A Comparative Empirical Analysis." British Journal of Political Science 18: 323-52.

Knutsen, Oddbjorn. 1989. "Cleavage Dimensions in Ten West-European Countries. A Comparative Analysis." Comparative Political Studies 21(4): 495-534.

Laver, Michael, ed. 2001. Estimating the Policy Position of Political Actors. London, New York: Routledge.

Laver, Michael y Ben Hunt. 1992. Policy and Party Competition. New York: Routledge, Chapman \& Hall.

Leiras, Marcelo. 2010. “Los procesos de descentralización y la nacionalización de los sistemas de partidos en América Latina." Política y gobierno 17(2): 205-41.

Mair, Peter. 2001. "Searching for the Positions of Political Actors. A Review of Approaches and a Critical Evaluation of Experts Serveys." En Estimating the Policy Position of Political Actors, editado por Michael Laver. London: Routledge, 10-30.

McKelvey, Richard D y Peter C Ordeshook. 1976. "Symmetric Spatial Games Without Majority Rule Equilibria." The American Political Science Review 70(4): 1172-84.

Mora y Araujo, Manuel. 1985. "La Naturaleza de la Coalicion Alfonsinista." En La Argentina Electoral. Buenos Aires: Editorial Sudamericana.

Mora y Araujo, Manuel. 2011. La Argentina Bipolar. Los vaivenes de la opinion publica (19832011). Buenos Aires: Sudamericana.

Ostiguy, Pierre. 2009a. "Argentina's Double Spectrum: Party System, Political Identities, and Strategies, 1944-2007." The Hellen Kellow Working Papers Series. University of Notre Dame.

Ostiguy, Pierre. 2009b. "The high and The Low in Politics: A Two-Dimensional Political Space for Comparative Analysis and Electoral Studies." The Helllen Kellog Working Papers Series. University of Notre Dame.

Ostiguy, Pierre. 2017. "Populism: A Socio-cultural Approach." En The Oxford Handbook of Populism, editado por Cristóbal Rovira Kaltwasser, Paul Taagart, Paulina Ochoa Espejo, y Pierre Ostiguy. Oxford: Oxford University Press, 73-97.

Reynoso, Diego. 2018. "Congruencia ideológica interprovincial de las coaliciones políticas nacionales." Revista de La SAAP 12 (1997): 99-130.

Rivas, Cristina. 2006. "Las dimensiones de la polarización en los partlamentos." En Políticas y Políticos en América Latina, editado por Manuel Alcántara Sáez. Madrid: Siglo XX, 215-254.

Saiegh, Sebastian M. 2009. "Recovering a Basic Space from Elite Surveys: Evidence from Latin America." Legislative Studies Quarterly 34 (1): 117-45.

Saiegh, Sebastian M. 2015. "Using Joint Scaling Methods to Study Ideology and Representation: Evidence from Latin America." Political Analysis 23 (3): 363-84. 
Sani, Giacomo y Giovanni Sartori. 1983. "Polarization, Fragmentation and Competition in Western Democracies." En Western European Party Systems, editado por H Daalder and P Mair. Beverly Hills: Sage.

Sarmiento, Domingo Faustino. 2010. Facundo o Civilización y barbarie en las pampas argentinas. Buenos Aires: Losada.

Schedler, Andreas. 2012. "Judgment and Measurement in Political Science." Perspectives on Politics 10 (01): 21-36.

Shepsle, Kennet A. y Ronald N. Cohen. 1990. "Multiparty Competition, Entry, and Entry Deterrence in Spatial Models of Election." En Advances in the Spatial Theory of Voting, editado por James Enelow and Melvin Hinch. Cambridge: Cambridge University Press, 12-45.

Steenbergen, Marco R. y Gary Marks. 2007. “Evaluating Expert Judgments.” European Journal of Political Research 46 (3): 347-66.

Stokes, Donald E. 1963. "Spatial Models of Party Competition." American Political Science Review 57 (2): 368-77.

Stokes, Susan. 2001. Mandates and Democracy. Neoliberalism by Surprise in Latin America. Cambridge: Cambridge University Press.

Svampa, Maristella. 1994. El dilema argentino: civilización o barbarie. De Sarmiento al revisionismo peronista. Buenos Aires: El cielo por asalto.

Tetreault, Mary y Pierre Ostiguy. 2014. “Operacionalizando el estilo político en las campañas electorales: Diferenciación 'alto-bajo' de los candidatos y sociología electoral del voto en la Argentina." Santiago de Chile: Pontificia Universidad Católica de Chile.

Vommaro, Gabriel y Sergio Morresi. 2016. "La ciudad nos une." En Hagamos Equipo: PRO Y La Construcción de La Nueva Derecha En Argentina, editado por Gabriel Vommaro y Sergio Morresi. Buenos Aires: Universidad Nacional de General Sarmiento.

Wiesehomeier, Nina. 2010. "The meaning of the Left-Right in Latin America: A Comparative View." University of Notre Dame.

Wiesehomeier, Nina y David Doyle. 2012. "Attitudes, Ideological Associations and the LeftRight Divide in Latin America." Journal of Politics in Latin America 4 (1): 3-33.

Zelaznik, Javier. 2001. "El sistema de partidos en Argentina a principios del siglo XXI." Iberoamericana 2 (32): 170-76.

Zubizarreta, Ignacio. 2014. Unitarios. Historia de la facción política que diseñó la Argentina moderna. Buenos Aires: Sudamericana.

Recibido: 10 de mayo de 2019

Aceptado: 23 de noviembre de 2019

Diego Reynoso. Investigador del CONICET y Profesor Titular de la Universidad de San Andrés, Doctor en Ciencia Política. Sus principales temas de investigación son los partidos, las elecciones y el comportamiento electoral, se especializa en el análisis de la opinión pública, los juicios de expertos y la ciencia de datos. Correo electrónico: dreynoso@udesa.edu.ar

Gonzalo Vronkistinos. Politólogo, ayudante de cátedra de la Universidad de San Andrés. Ha sido asesor en los Ministerios de Energía y en Jefatura de Gabinete de Ministros de la República Argentina y se ha desempeñado como consultor en distintos proyectos de investigación. Correo electrónico: gvronkistinos@udesa.edu.ar 